\title{
XPM-INDUCED INTENSITY NOISE IN WDM COMPENSATED TRANSMISSION SYSTEMS
}

\author{
Giovanni Bellotti, Matteo Varani, Cristian Francia, and Alberto Bononi
}

Dipartimento di Ingegneria dell'Informazione Viale delle Scienze, I-43100 Parma, Italy phone: +39-521-905-750, fax: +39-521-905-758, (e-mail: bononi@tlc.unipr.it)

Abstract: A new linear model of the XPM-induced intensity noise is used for a fast evaluation of the system degradation at the end of several types of dispersion compensated transmission systems.

\begin{abstract}
Introduction
In dispersion compensated systems the intensity distortion induced by the interplay between cross-phase modulation (XPM) and group velocity dispersion (GVD) can be a primary cause of transmission degradation. Like any phase noise, XPM generates intensity distortion at the end of a dispersive fiber [1], but because of its distributed generation, compensation cannot perfectly undo such distortion. In this paper we give the expression of a linear filter that accounts for the intensity/cross-phase/intensity conversion (IM/XPM/IM) at the end of any type of dispersion compensated system. This allows a fast computation of the XPMinduced intensity noise at the receiver, without resorting to very long simulations, so that it becomes a very useful tool in the design of terrestrial long-haul wavelength division multiplexed (WDM) transmission systems.
\end{abstract}

\section{Theory}

We consider an N-channel WDM system propagating over a transmission fiber of length $\mathrm{L}_{1}$ followed by a compensating fiber of length $\mathrm{L}_{2}$. In [3] we gave an explicit expression of an IM/XPM filter in the general case of dispersion compensated systems, capable of very well predicting the reference signal phase. In presence of chromatic dispersion, the XPM contribution generated at a given point along the fiber is converted by GVD into intensity modulation during the propagation from that point to the end of the fiber [1]. Assuming all the contributions of each infinitesimal segment add up to build the total intensity noise, the global XPMinduced relative intensity variation at the end of the span is:

$$
\frac{\Delta \mathrm{P}_{\mathrm{s}}(\omega)}{\left\langle\mathrm{P}_{\mathrm{s}}\right\rangle}=-2 \gamma \sum_{\mathrm{p} \neq \mathrm{s}} \mathrm{P}_{\mathrm{p}}(0, \omega) \mathrm{H}_{\mathrm{sp}}^{\mathrm{IM}}(\omega)
$$

where $\langle\mathrm{Ps}\rangle$ is the time averaged input signal power, $\gamma$ is the fiber nonlinear coefficient, $\mathrm{P}_{\mathrm{p}}(0, \omega)$ the Fourier transform of the p-th input channel power, and $\mathrm{H}_{\mathrm{sp}}{ }^{\mathrm{IM}}(\omega)$ is the total $\mathrm{IM} / \mathrm{XPM} / \mathrm{MM}$ filter for the $\mathrm{p}$-th interferer. In the special case of perfect compensation at channel $\mathrm{s}$, and neglecting nonlinear effects in the compensating fiber, such filter simplifies to:

$$
\begin{aligned}
H_{\mathrm{sp}}^{\mathrm{IM}}(\omega)= & j\left(\frac{1-\exp \left[\left(-\alpha_{1}+\mathrm{j}\left(\mathrm{d}_{\mathrm{sp}} \omega-\Psi \omega^{2}\right)\right) \mathrm{L}_{1}\right]}{\alpha_{1}-\mathrm{j}\left(\mathrm{d}_{\mathrm{sp}} \omega-\Psi \omega^{2}\right)}\right) \\
& -\left(\frac{1-\exp \left[\left(-\alpha_{1}+j\left(\mathrm{~d}_{\mathrm{sp}} \omega+\Psi \omega^{2}\right)\right) \mathrm{L}_{1}\right]}{\alpha_{1}-j\left(\mathrm{~d}_{\mathrm{sp}} \omega+\Psi \omega^{2}\right)}\right)
\end{aligned}
$$

where $\Psi=\lambda^{2} D_{1} / 4 \pi c, \lambda$ being the central wavelength and $c$ the light velocity; $D_{1}, \alpha_{1}$, and $d_{s p}$ are the chromatic disper- sion, the attenuation coefficient, and the walk-off parameter between channels $s$ and $p$ [2], respectively, of the transmission fiber. Equation (1) can be easily extended to the general case of a system composed of $\mathbf{M}$ amplified and compensated links, without neglecting the compensation fiber contributions [4].

Fig. 1: Squared magnitude of the IM/XPM/IM filter. Channel separation $0.8 \mathrm{~nm}$

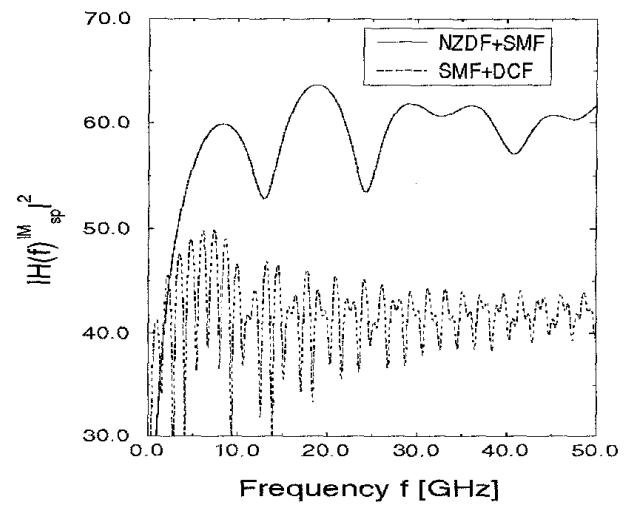

As an example, Fig.1 gives the squared magnitude of $\mathrm{H}_{\mathrm{sp}}{ }^{\mathrm{IM}}(\omega)$ for a single compensated span, with perfect compensation at channel $s$, for two different compensating schemes. In the first scheme (solid line), the transmission fiber is a non-zero dispersion fiber (NZDF), with $D_{1}=-2$ $\mathrm{ps} / \mathrm{nm} / \mathrm{km}, \mathrm{L}_{1}=85 \mathrm{~km}$, and the compensating fiber is a single mode fiber (SMF) with $D_{2}=17 \mathrm{ps} / \mathrm{nm} / \mathrm{km}$ and $\mathrm{L}_{2}=10 \mathrm{~km}$. Other parameters common to the two fibers are the dispersion slope $D^{\prime}=0.07 \mathrm{ps} / \mathrm{km}^{\prime} / \mathrm{nm}^{2}, \gamma=2.35 \mathrm{~W}^{-1} \mathrm{~km}^{-1}$ and $\alpha=0.21 \mathrm{~dB} / \mathrm{km}$. In the second scheme (dashed line), the transmission fiber is a SMF as above, with $\mathrm{L}_{1}=57 \mathrm{~km}$, and a dispersion compensating fiber (DCF) with $\mathrm{D}_{2}=-95$ $\mathrm{ps} / \mathrm{nm} / \mathrm{km}, \mathrm{L}_{2}=10.2 \mathrm{~km}, D^{\prime}=0.07 \mathrm{ps} / \mathrm{km}^{\prime} / \mathrm{nm}^{2}, \gamma_{2}=6 \mathrm{~W}^{-1} \mathrm{~km}^{-1}$, $\alpha_{2}=0.6 \mathrm{~dB} / \mathrm{km}$ is used for compensation. We clearly see that the XPM filtering action is more effective in the SMF+DCF scheme, where the walk-off parameter is larger $[2,3]$.

Results

We used the theoretical model to obtain the XPM noise variance on channel $s$ at the receiver as:

$$
\sigma_{\mathrm{t}}^{2}=4 \gamma^{2}\left\langle\mathrm{P}_{\mathrm{s}}\right\rangle^{2} \int_{-\infty}^{\infty} \mathrm{S}_{\mathrm{p}}(0, \omega)\left|\mathrm{H}_{\mathrm{sp}}^{\mathrm{IM}}(\omega)\right|^{2} \mathrm{~d} \omega
$$

where $S_{p}(0, \omega)$ is the spectrum of the interferer power on channel p. For a single span perfectly compensated system, 
Fig. 2 shows $\sigma_{t}^{2}$ versus the bit rate $\mathrm{R}$ of the $\mathrm{p}$ channel for both of the previous compensation schemes.

Fig. 2: $\sigma_{t}^{2}$ versus bit rate.

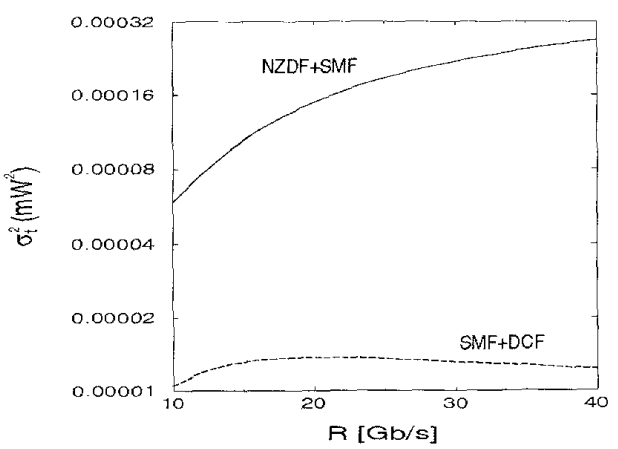

The peak power of both channel $\mathrm{s}$ and $\mathrm{p}$ is $5 \mathrm{dBm}$, the channel spacing is $\Delta \lambda=0.8 \mathrm{~nm}$, and channel $\mathrm{p}$ is on-off keying (OOK) modulated with nonreturn-to-zero (NRZ) raised cosine pulses (roll-off 0.8 ). The variance is larger for the NZDF+SMF scheme, being the IM/XPM/IM filter stronger in this case, as confirmed by Fig.1. The variance also grows monotonically in the range shown, while in the SMF+DCF scheme the variance has a maximum and then decreses for higher bit rates. This is a direct consequence of the shape of the IM/XPM/IM filter plotted in Fig.1.

\section{Fig. 3: $\sigma_{t}^{2}$ versus degree of compensation} for two different systems.

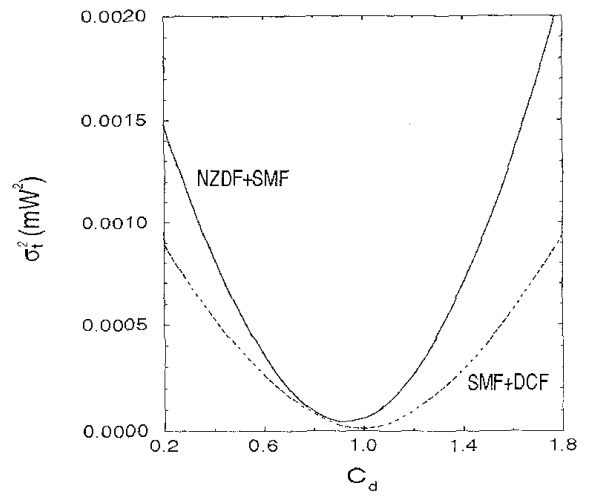

Fig. 3 shows a plot of $\sigma_{t}^{2}$ versus the degree of dispersion compensation, defined as $C_{d}=1 D_{2} L_{2} / D_{1} L_{1}$. In this case we kept $L_{1}$ fixed and varied the length $L_{2}$. For a single span and the power levels used, a slight undercompensation $\left(\mathrm{C}_{\mathrm{d}}<1\right)$ minimizes the XPM-induced intensity distortion for the SMF+DCF scheme, while more undercompensation is needed to optimize the NZDF+SMF scheme.

Simulations based on the split-step Fourier method (SSFM) were carried out to verify the goodness of the theoretical predictions. Three WDM channels, with $5 \mathrm{dBm}$ peak power per channel and $\Delta \lambda=0.8 \mathrm{~nm}$ spacing, with a $C W$ probe central channel and two $10 \mathrm{~Gb} / \mathrm{s}$ modulated edge channels were propagated along a chain of span-by-span compensated and amplified links. The compensation schemes were: 1) $\mathrm{NZDF}+\mathrm{SMF}$ and 2) SMF+DCF as described before; and 3) DCF+SMF, where we swapped the position of the transmission and compensating fiber in scheme 2). Exact compensation was achieved at the $\mathrm{CW}$ channel.
We consider the power variation induced by XPM on the probe channel and we define the sample variance as $\sigma^{2}$ $=(1 / N) \sum_{k=1}{ }^{N}\left[P_{s}\left(k T_{0} / N\right)-\left\langle P_{s}>\right]^{2}\right.$ where $P_{s}$ is the channel power and $\mathrm{N}=8192$ is the number of samples in the observation time $T_{0}$ corresponding to 128 bits. In Fig. 4 we plot $\sigma$ versus the number of compensated spans both for simulations (solid line) and for our model (diamonds). As we see from the figure, the IM/XPM/IM theoretical model well predicts the output probe power variations. Note that, for a single simulation with $M=30$ spans, the SSFM takes 10 hours on a SUN workstation, while our linear model takes $5 \mathrm{~min}$. Note also that the DCF+SMF system, which has been shown to be the best dispersion map in the case of single channel propagation [5], gives the worst performance with respect to XPM effects.

Fig. 4: $\sigma$ versus number of spans in three different maps. Solid: simulation; Diamonds: theory.

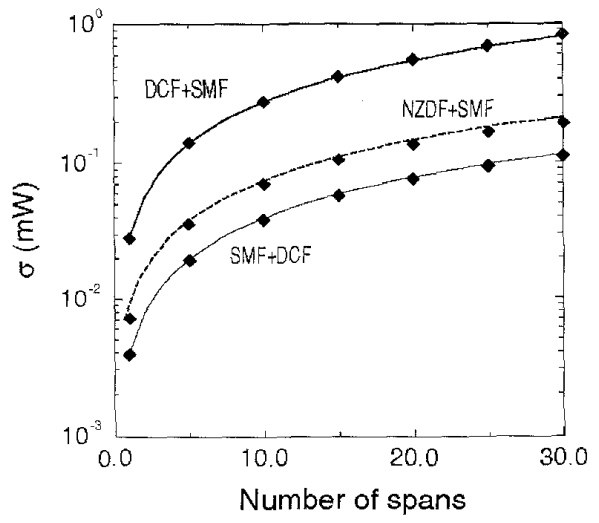

\section{Conclusion}

A linear filter that accurately describes the XPM-induced intensity noise in WDM systems is presented. Our IM/XPM/IM theoretical model gives accurate predictions of the output intensity distortions over terrestrial transmission distances, so that it can be used as a quick design tool.

This work was supported by the European project INCODC No.950959 'DAWRON', and by a grant from CSELT .

\section{References}

11/ J. Wang, and K. Petermann, lEEE J. Lightwave Technol., vol.10, pp.96-100, Jan. 1992.

12/ T. Chiang, N. Kagi, M. E. Marhic, and L. Kazovsky, IEEE J. Lightwave Technol, vol.14, pp.249-260, Mar. 1996

13/ A. Bononi, C. Francia, and G. Bellotti, Optical Fiber Technol., to appear.

14/ G. Bellotti, M. Varani, C. Francia, A. Bononi, CISS '98, paper TP4.2, Princeton, NJ, March 1998.

15/ D. M. Rothnie, and J. E. Midwinter, Elecron. Lett., vol. 32, pp. 1907-1908, Sept. 1996. 